\title{
Supervivencia y crecimiento de un árbol nativo maderable bajo diferentes coberturas de dosel en el Bosque Atlántico, Misiones, Argentina
}

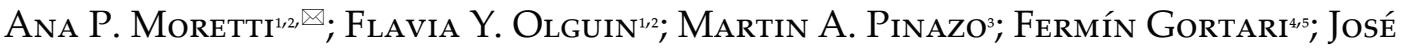 \\ VERA BAHIMA ${ }^{1,2,6}$ \& CORINA GRACIANO ${ }^{1,2,6}$ \\ ${ }^{1}$ INFIVE (CONICET - Universidad Nacional de La Plata). La Plata, Buenos Aires, Argentina. ${ }^{2}$ CCT CONICET La Plata. La \\ Plata, Buenos Aires, Argentina. ${ }^{3}$ INTA EEA Montecarlo. Montecarlo, Misiones, Argentina. ${ }^{4}$ Facultad de Ciencias Forestales, \\ Universidad Nacional de Misiones. Misiones, Argentina. ${ }^{5}$ CCT CONICET Nordeste. Corrientes, Argentina. ${ }^{6}$ Facultad de \\ Ciencias Agrarias y Forestales, Universidad Nacional de La Plata. La Plata, Buenos Aires, Argentina.
}

\begin{abstract}
Resumen. En la selva, la dinámica de disturbios de origen natural o humano genera cambios en la cobertura arbórea y en la cantidad y la calidad de luz que llega al sotobosque. Los cambios debidos a los disturbios representan un continuo de condiciones microambientales que garantizan la coexistencia de las especies. El conocimiento de los requerimientos de las especies y la evaluación de los microambientes óptimos para su supervivencia y crecimiento permite planificar estrategias de manejo a fin de recuperar áreas forestales degradadas. Trabajamos en un bosque nativo ubicado en San Antonio, Misiones, con una especie nativa de alto valor ecológico y maderero: Cabralea canjerana (Vell.) Mart. (cancharana). El objetivo fue evaluar el establecimiento de esta especie bajo diferentes intensidades de cobertura de dosel del bosque. Se establecieron 2 ensayos: uno en macetas, en el que se evaluó la supervivencia y el crecimiento de plantas expuestas a dos condiciones de cobertura natural contrastantes, y otro con plantación a campo en un gradiente de cobertura generado por claros naturales. En ambos ensayos, más de $90 \%$ de las plantas de cancharana se establecieron inicialmente en un gradiente muy amplio de cobertura. En macetas, las plantas sin cobertura (sol pleno) fueron afectadas por heladas durante el invierno. A 4 años de realizada la plantación en los claros, la supervivencia fue de $70 \%$ y la mayor mortalidad se registró en microambientes con menor incidencia de luz. Concluimos que se puede enriquecer el bosque nativo con cancharana, que el microambiente no afecta la supervivencia durante el primer año, pero que el crecimiento posterior es mayor en posiciones con menor cobertura. La especie tolera cambios abruptos de cobertura, por lo que la limpieza del sotobosque y la apertura de claros benefician su crecimiento.
\end{abstract}

[Palabras clave: sombra, restauración ecológica, cancharana, Selva Paranaense]

ABstRACT. Survival and growth of a timber tree under different canopy coverages in the Atlantic Forest, Misiones, Argentina. The natural dynamic of the rainforest generates changes in the canopy coverage and, consequently, in the quality and quantity of light that reaches the understory. Similar effects can be achieved by the anthropogenic selective extraction of trees. The microsites produced by the disturbances represent a continuum of environmental conditions that ensures the coexistence of many species. The knowledge of the requirements of the species and the evaluation of the optimal microenvironments to plant growth are useful to build tools to restore degraded areas. We worked in a native rainforest in San Antonio, Misiones, Argentina, focused in a species that has high timber and ecological value: Cabralea canjerana (Vell.) Mart. (cancharana). The aim was to evaluate the establishment of cancharana under different coverage conditions. Two experiments were carried out: a pot experiment in which survivor and growth under two extreme natural coverage were evaluated, and a field experiment in which cancharana was planted in a gradient of coverage produced by natural gaps. In both experiments, more than $90 \%$ of the cancharana plants were initially established in a wide gradient of coverage. In the pot experiment, plants without forest coverage (full sun) were damaged by the frost in winter. Four years after the plantation, $70 \%$ of the plants survived in the field experiment and mortality was observed mostly in microenvironments with lower light incidence. We conclude that cancharana can establish and survive in many microenvironmental conditions within the rainforest, it acclimates in few months to sudden changes in coverage and, one year after planting, its growth arises with higher availability of light. The species tolerates sudden changes in coverage, therefore the remove of the understory or gap opening stimulate cancharana growth.

[Keywords: shade, ecological restoration, cancharana, rainforest]

Editor asociado: Martín Núñez

$\bar{\square}$ anapaulamoretti@agro.unlp.edu.ar
Recibido: 30 de Junio de 2018

Aceptado: 31 de Dicieubre de 2018 


\section{INTRODUCCIÓN}

Los bosques subtropicales son formaciones mutiespecíficas y multietáneas, es decir que conviven individuos de diferentes especies y edades, con gran riqueza de especies. Como los ambientes son muy heterogéneos, se genera una gran diversidad de nichos ecológicos que forman un continuo de microambientes en los que se desarrollan las especies (Wright et al. 2003). Si bien hay muchos factores ambientales que definen estos microambientes en el bosque, la luz es uno de los más importantes, ya que interviene de forma directa con los procesos de regeneración, germinación y crecimiento de las plantas (Chazdon et al. 1996; Montagnini and Jordan 2005). La luz llega a los diferentes estratos del bosque en diferentes calidades e intensidades. La radiación directa que llega al dosel es absorbida principalmente por los estratos superiores e intermedios. Sin embargo, una pequeña cantidad de luz atraviesa la porosidad del dosel y llega al sotobosque. A medida que la luz atraviesa la copa de los árboles, su intensidad disminuye y se enriquece proporcionalmente en rojo lejano, ya que la luz roja es absorbida de manera preferencial por las hojas de las plantas. Por su parte, el rojo lejano es absorbido en menor proporción, atraviesa las hojas y se refleja, por lo cual llega a los estratos más bajos (Fitter and Hay 2002). De este modo, la relación rojo: rojo lejano varía según la cobertura existente. Este cociente es fundamental en la inducción de la respuesta morfológica y fisiológica de las plantas a la cobertura (e.g., la elongación de entrenudos hacia la luz, el aumento en la concentración de clorofilas y la superficie foliar en plantas que crecen a la sombra) (Dudley and File 2007). La formación de claros en la selva cambia significativamentela cobertura arbórea, favorece la llegada de luz directa al sotobosque y altera las condiciones microambientales del sitio (Denslow 1987; Amir 2012; Garbarino et al. 2012). Las especies coexisten en el continuo de microambientes ya que tienen diferentes requerimientos de recursos y plasticidad fenotípica para responder a esos cambios en el microambiente. Si bien hay especies estrictamente tolerantes a la sombra que se desarrollan en el sotobosque (Welden et al. 1991; Montgomery and Chazdon 2002), y otras que requieren luz para germinar (poseen semillas fotoblásticas) o elevada radiación solar para crecer (pioneras), la gran mayoría de las especies tropicales y subtropicales son consideradas intermedias en cuanto a los requerimientos de luz. Dichos requerimientos varían según el estadío de desarrollo en que se encuentren los individuos (Wright et al. 2003; Valladares et al. 2004). Los claros en la selva se producen de forma natural por la caída de grandes ramas de árboles, por la muerte de uno o más ejemplares o por otras causas como el volteo por el viento (Runkle 1992). Es uno de los eventos más importantes para que se regeneren y establezcan las especies pioneras de rápido crecimiento, siempre y cuando haya semillas disponibles y sean dispersadas. También estimulan el crecimiento de aquellas especies de la sucesión secundaria que inicialmente se establecen en el sotobosque, pero requieren mayor cantidad de luz para crecer y llegar a los estratos superiores. Por otro lado, en los claros, las especies de rápido crecimiento dominan el sotobosque y generan una elevada competencia por los recursos. La extracción selectiva de ejemplares maderables es la principal causa antrópica de generación de claros. Los factores más importantes que definen los microambientes en el bosque son la frecuencia y la distribución espacial de los claros, y el tamaño y la orientación de cada claro (Amir 2012; Garbarino et al. 2012). Asimismo, el relieve, el tamaño de los árboles que rodean al claro y la presencia de especies remanentes dentro de éstos también determinan las condiciones microambientales. En las selvas tropicales, el tamaño de los claros naturales puede variar desde menos de $10 \mathrm{~m}^{2}$ hasta más de $400 \mathrm{~m}^{2}$, mientras que en ambientes templados pueden medir entre $20 \mathrm{~m}^{2}$ y $5000 \mathrm{~m}^{2}$ (Denslow 1987; Coates 2002; De Lima and De Moura 2008).

La formación de un claro en el bosque generará un continuo de microambientes a partir del centro (donde la cobertura es menor) hasta los límites del claro (donde aumenta por efecto de la vegetación del borde). Un gradiente microambiental puede generar diferentes respuestas en supervivencia y crecimiento. Es esperable que las especies heliófitas sobrevivan y crezcan más en microambientes más iluminados (Fitter and Hay 2002). Sin embargo, no hay una evidencia clara de que exista una relación simple entre el tamaño de un claro y el éxito de la regeneración de las especies tolerantes e intolerantes a la sombra (posiblemente porque la interacción con otros factores como la disponibilidad de luz, de agua y de nutrientes afecta el éxito de la regeneración) (Gasser et al. 2010). La supervivencia durante el primer año de vida de nueve especies tropicales, definidas $a$ priori como especies demandantes de luz, fue 
mayor en microambientes más sombreados que en los más iluminados. Sin embargo, el crecimiento en altura y en materia seca fue mayor cuando la disponibilidad de luz también lo fue (Yang et al. 2011). Por lo tanto, en estas especies, la cobertura de dosel contribuye a la supervivencia inicial, pero el crecimiento posterior se asocia de forma positiva con menores niveles de cobertura. El conocimiento de los ambientes lumínicos generados por la dinámica natural de la selva y los requerimientos de las especies nos permitirán entender el funcionamiento de los ecosistemas y producir información para idear estrategias de manejo a fin de restaurar áreas degradadas en los bosques. La selva misionera, que pertenece al Bosque Atlántico, sufrió alteraciones continuas en su estructura y composición, con una consecuente degradación de los recursos y la pérdida de funcionalidad del ecosistema. Las principales causas fueron la extracción selectiva de árboles de alto valor maderero y el cambio de uso de la tierra para la producción de alimentos (Di Bitetti et al. 2003). La gran mayoría de los bosques sufrieron intervenciones que derivaron en ambientes perturbados. Estos ambientes podrían recuperarse solos; sin embargo, es posible acelerar los procesos de recuperación mediante su intervención a través de la restauración ecológica.

La restauración ecológica tiene como uno de sus objetivos relevantes recuperar la funcionalidad de los ecosistemas en el tiempo. El enriquecimiento es una de las herramientas para restaurar; consiste en incorporar especies nativas de valor ecológico en sitos de bosques degradados sin alterar lo ya existente (Montagnini et al. 2006). Sin embargo, al momento de implementar las prácticas de enriquecimiento en el bosque nativo, la falta de información acerca de cómo, dónde y cuándo realizar la acción de enriquecimiento o restauración por lo general impide tomar decisiones fundamentadas. Por lo tanto, estudiar la autoecología de las especies y evaluar los sitios donde llevar a cabo los procesos de restauración es muy importante para poder proponer prácticas de manejo y restauración. Es decir, disponer de evaluaciones científicas para encontrar respuestas a diversas preguntas prácticas: dónde plantar, con qué especies, con qué semillas, en qué época o qué características microambientales de los sitios favorecen el establecimiento y crecimiento, entre otras (Morales et al. 2018).
Enesteestudiose trabajócon Cabralea canjerana (Vell) Mart. (cancharana), una especie arbórea nativa de la selva misionera. C. canjerana es una espacie nativa de la Selva Paranaense y posee un alto valor ecológico y maderero. Su área de distribución se extiende desde Costa Rica hasta el sur de Brasil, el este de Paraguay y el norte de la Argentina (Schüssler 2006). Se observó que la cancharana se regenera naturalmente en diversos ambientes, bajo bosque denso, capueras y áreas abiertas (Cargnelutti Filho et al. 2012; Aimi 2014). Se la clasificó como una especie secundaria tardía dentro de las etapas de sucesión de la selva, y se la considera tolerante a la sombra en las etapas juveniles (Carvalho 1996). No existen evaluaciones que corroboren esta clasificación. Posee abundante producción de semillas, germinación y establecimiento inicial. Sin embargo, sólo algunos individuos llegan a los estratos intermedios y superiores del dosel (Ríos et al. 2008; Moscovich et al. 2010). En la etapa adulta, la cancharana alcanza hasta $30 \mathrm{~m}$ de altura y 1.5 m de diámetro (Carvalho 1996; Tortorelli 2009). Por otro lado, la técnica de producción en vivero es conocida y se pueden producir plantines de cancharana con facilidad (González and Barth 2013).

El objetivo de este trabajo fue evaluar el establecimiento de la cancharana bajo diferentes intensidades de cobertura de dosel. Se busca responder las siguientes preguntas: ¿Cómo afecta el microambiente de plantación la supervivencia y el crecimiento de la cancharana? ¿Dónde conviene plantar? ¿Cancharana tolera el cambio abrupto de cobertura (producido, por ejemplo, por la limpieza del sotobosque o por la apertura de un claro)? Nuestra hipótesis postula que, dado que fue descripta como una especie tolerante a la sombra, el crecimiento será mayor en los bordes del claro que en el centro, y que el cambio abrupto de cobertura puede producir estrés hídrico o lumínico que se reflejará en una disminución del crecimiento.

\section{Materiales y Métodos}

Para este trabajo se realizaron 2 ensayos. El primero fue en macetas, en condiciones naturales de cobertura. Se llevó a cabo en 2 etapas: en la primera, se evaluó el crecimiento y supervivencia de las plantas jóvenes de cancharana en ambientes contrastantes de cobertura (canopeo denso y sol pleno) durante 18 meses. En la segunda, se evaluó el cambio abrupto de la cobertura. 
El segundo ensayo fue de plantación a campo en claros del bosque nativo. Se realizó para evaluar la supervivencia y crecimiento de plantas de cancharana plantadas en un gradiente lumínico generado por claros. Para esto se seleccionaron claros naturales en el bosque nativo, que representaron un gradiente de cobertura y se plantaron grupos de plantas en diferentes posiciones dentro del claro, que se describen en el apartado "Ensayo en claros".

\section{Área de estudio}

Los ensayos fueron llevados a cabo en el predio del Campo Anexo Manuel Belgrano (260'ㄷ․ $91^{\prime \prime} \mathrm{S}-53^{\circ} 46^{\prime} 14.15^{\prime \prime} \mathrm{O}$; $552 \mathrm{~m}$ s. n. m.), dependiente del INTA EEA Montecarlo, San Antonio, Misiones, Argentina. Los sitios utilizados se ubicaron en un bosque remanente de 5 ha que fue aprovechado por última vez hace más de 50 años. La temperatura media anual es de $20.4{ }^{\circ} \mathrm{C}$, la mínima absoluta histórica de julio es $-7^{\circ} \mathrm{C}$ y la máxima absoluta histórica de julio $30.5^{\circ} \mathrm{C}$, la temperatura máxima absoluta histórica de enero es 36.5 ${ }^{\circ} \mathrm{C}$ y la temperatura mínima absoluta histórica de enero $8.9^{\circ} \mathrm{C}$. Las precipitaciones rondan los $2100 \mathrm{~mm} /$ año, distribuidas de manera homogénea a lo largo del año. El promedio de días con lluvia para los últimos 34 años es de 116 días/año (datos climáticos del lugar donde se instaló el ensayo en macetas, a 1500 $\mathrm{m}$ del ensayo en claros).

\section{Ensayo en macetas}

Las semillas utilizadas para hacer los plantines fueron recolectadas de árboles dentro del Campo Anexo Manuel Belgrano. Las plantas crecieron en invernáculo con media sombra hasta el momento del trasplante. En la primavera de 2012, las plantas de cancharana de 8 meses de edad, con un promedio de 15 $\mathrm{cm}$ de altura, fueron repicadas a macetas de 7 L con mezcla de suelo y sustrato de corteza de pino, y colocadas bajo dos coberturas contrastantes: 34 plantas fueron colocadas a sol pleno (nivel de tratamiento sol pleno; $S$ ) y a 68 plantas se las ubicó bajo canopeo denso en el bosque (nivel de tratamiento canopeo denso; C). En la primavera de 2013, la mitad de las macetas del nivel de tratamiento canopeo fueron sometidas a un cambio abrupto de la cobertura al pasarlas a sol pleno (nivel de tratamiento shock lumínico; SL), mientras que la otra mitad continuó bajo canopeo denso hasta los 18 meses. Las mediciones se realizaron a los 6, 12 y 18 meses de instalado el ensayo. Las plantas de sol, además de la lluvia, recibieron riego por aspersión una o dos veces por día según la época del año y la frecuencia de lluvias. Las plantas de canopeo recibieron sólo agua de lluvia. El potencial hídrico y la conductancia estomática al mediodía de las plantas de canopeo fue mayor que el de las plantas de sol, por lo que no sufrieron estrés por falta de agua (datos no mostrados).

\section{Ensayo en claros}

En septiembre de 2014 se plantaron 162 plantas de cancharana de 10 meses de edad y una altura media de $14 \mathrm{~cm}$, en diferentes posiciones de 3 claros del bosque nativo. Los claros seleccionados tenían 68, 119 y $172 \mathrm{~m}^{2}$ de área. Estos tamaños son muy frecuentes en los bosques tropicales y subtropicales (Denslow 1987; De Lima and De Moura 2008). Las diferentes posiciones dentro del claro implican que las plantas estuvieron expuestas a un gradiente de cobertura, representando sitios que recibieron mayor disponibilidad de luz (centros de los claros) hasta sitios donde la disponibilidad de luz era muy escasa (debajo del canopeo). En cada claro, las plantas se ubicaron en grupos de 9 plantas en 5 posiciones dentro de cada claro (centro, norte, sur, este, oeste) y en una posición dentro del bosque para simular la condición de cobertura densa (bosque). Las 9 plantas en cada posición estaban distanciadas $2 \mathrm{~m}$ entre sí, ordenadas en un cuadrado de $3 \times 3$ plantas. El día que se realizó la plantación, se limpió con machete la vegetación herbácea y arbustiva para poder caminar y plantar. Se midió longitud norte-sur y este-oeste a fin de identificar el centro del claro. Las posiciones norte y sur se plantaron equidistantes del centro, al igual que las este y oeste. Como los claros no son circulares, la distancia entre las parcelas norte y sur no fue la misma que la distancia entre las parcelas este y oeste, ni fue la misma en todos los claros. Las parcelas se plantaron desde la proyección de copas del borde; la tercera fila llegó a $6 \mathrm{~m}$ hacia el interior del bosque. La plantación se realizó haciendo un pozo con un saracuá, de forma que entrara en ancho y profundidad el pan de tierra del tubete de $5 \mathrm{~cm}$ de diámetro y $15 \mathrm{~cm}$ de altura. No se eliminó ni removió el mantillo, más allá de la alteración que ocasionó realizar el pozo. No se realizó riego de asiento. Los registros se realizaron en la primavera y otoño durante 3 años, desde la instalación de los ensayos. Los claros se limpiaron 2 veces al año, en primavera y otoño, unos días antes de 
realizar las mediciones. La limpieza consistió en el macheteado de herbáceas alrededor de cada planta; esta práctica es común, ya que muchas especies pioneras colonizan rápidamente el sitio y compiten por los recursos. Si bien esto altera la competencia en el momento de la medición, no es posible ingresar a medir a los claros si no se realiza el control de estas herbáceas.

\section{Cobertura}

Para caracterizar el gradiente de cobertura en el que se establecieron las plantas de ambos ensayos se midieron diferentes variables. La radiación fotosintéticamente activa (PAR) $\left(\mu \mathrm{mol} \cdot \mathrm{m}^{-2} \cdot \mathrm{s}^{-1}\right)$ se midió con ceptómetro (Cavadevices, Buenos Aires, Argentina), arriba de cada planta, al mediodía. La relación rojo:rojo lejano (R/RL) se midió con un espectrorradiómetro Red Tide USB 650 (Ocean Optics, Dunedin, Florida, USA), a $670 \mathrm{~nm}$ y $730 \mathrm{~nm}$, respectivamente. Por otro lado, en el ensayo de los claros se tomaron fotos hemisféricas con lente de ojo de pez en el centro de cada parcela (posición por claro), para lo cual se ubicó la cámara a $1 \mathrm{~m}$ de altura sobre el piso (fish-eye de $180^{\circ}$ Nikon FC-E9 incorporado al sistema auto-nivelante SML6, Delta T Devices, y cámara digital Nikon Coolpix 8400 adaptada a la lente mediante adaptador UR-E16 de Nikon). Las fotos fueron tomadas antes de la salida del sol para evitar que los rayos de sol directo distorsionaran las estimaciones. Luego, con el software GLA (Gap Light Analyzer) se estimó la media diaria de luz directa transmitida total $\left(\mathrm{mol} \cdot \mathrm{m}^{-2}\right.$.día $\left.{ }^{1}\right)$ y el porcentaje de canopeo abierto en cada posición por claro (Frazer et al. 1999).

\section{Supervivencia y crecimiento}

Para evaluar la supervivencia se consideró que la planta estaba muerta si la totalidad de la parte aérea (vástago) estaba muerta o no estaba. En algunos casos, plantas registradas como muertas rebrotaron desde la raíz, por lo que en la siguiente medición se las consideró vivas. Se reportó la supervivencia a los 6, 12 y 18 meses para el ensayo en macetas, y a los 6,12, 18,36 y 44 meses para el ensayo de los claros. Para evaluar el crecimiento, en simultáneo con las evaluaciones de supervivencia se estimó la altura $(\mathrm{cm})$ usando cinta métrica.

\section{Peso seco y partición de materia seca}

Para evaluar los posibles cambios morfológicos de plantas de cancharana en respuesta a diferentes ambientes se midió la partición de materia seca en el ensayo en macetas, a los 6, 12 y 18 meses. Para esto se procesaron 6 plantas completas por tratamiento presente en cada muestreo. Las plantas se separaron en tallo, hojas, raíces finas y raíces gruesas (raíz pivotante), y se llevaron a estufa hasta peso constante.

\section{Visualización de los resultados y análisis estadístico}

Para analizar la diferencia de pesos seco (ensayo en macetas) se realizó un ANOVA de un factor con 2 ó 3 niveles de luz, por separado para cada fecha de medición. Esta decisión se tomó porque el tratamiento sol no estuvo presente a los 12 meses y el tratamiento shock lumínico estuvo presente sólo a los 12 y 18 meses. Para cada fecha, si el ANOVA fue significativo $(P<0.05)$, se realizó el análisis de comparación de medias con el método de Duncan con $P<0.05$. Los datos fueron analizados con el software InfoStat (Casanoves et al. 2012). Para el ensayo en los claros se realizó un gráfico 3D tomando como ejes: 1) la altura de las plantas en los diferentes meses de medición ( $\mathrm{cm}), 2$ ) la luz transmitida total $\left(\mathrm{mol} \cdot \mathrm{m}^{-2} \cdot\right.$ día $\left.^{-1}\right)$, integrada para todo el año por posición en cada claro, y 3) el tiempo (meses). Con este gráfico se puede apreciar el crecimiento en altura de las plantas en las diferentes condiciones de luz a lo largo del tiempo. Por otro lado, se realizó un análisis de regresión no lineal a dos tramos. Se tomó como variables dependientes las alturas medias de las plantas en las diferentes categorías de luz transmitida total [menor a 5 mol.m ${ }^{-2}$.día ${ }^{-1}$ (luz <5), entre 5 y 10 mol.m ${ }^{2}$.día ${ }^{-1}$ (luz 5-10), entre 10 y 15 mol.m - $^{-2}$. día ${ }^{-1}$ (luz 10-15) y mayor a 15 mol.m ${ }^{-2}$.día $\left.{ }^{-1}(\mathrm{luz}>15)\right]$. Como variable regresora se tomó el tiempo en meses. Además, se calculó la distribución de frecuencias relativas de plantas vivas y muertas por clase de PAR. El rango de las clases de PAR es de $47 \mu \mathrm{mol} . \mathrm{m}^{-2} . \mathrm{s}^{-1}$.

\section{Resultados}

\section{Cobertura}

El PAR medido al mediodía en el ensayo en macetas, en el cual se establecieron 2 condiciones de cobertura contrastantes, fue de $1700 \mu \mathrm{mol} . \mathrm{m}^{-2} \cdot \mathrm{s}^{-1}$ en el tratamiento de sol y $\sim 200 \mu \mathrm{mol} . \mathrm{m}^{-2} . \mathrm{s}^{-1}$ en el tratamiento de canopeo denso. Por otro lado, la relación rojo: rojo lejano fue 1.04 a sol pleno y 0.75 bajo el canopeo denso (Tabla 1). 
Tabla 1. Relación rojo-rojo lejano (R:RL) en el ensayo en macetas, a lo largo del día, en plantas expuesta al sol y bajo el canopeo. En el ensayo en claros del bosque nativo, las mediciones se realizaron al mediodía y la relación R:RL se reporta por rango de luz transmitida total, estimada con las fotos hemisféricas [menor a $5 \mathrm{~mol}^{-2} \mathrm{~m}^{-2}$.día ${ }^{-1}(<5)$, entre 5 y 10 mol.m ${ }^{-2}$.día ${ }^{-1}$ (5-10), entre 10 y 15 mol.m ${ }^{-2}$.día ${ }^{-1}(10-15)$ y mayor a 15 mol.m $\mathrm{m}^{-2}$.día $\left.{ }^{-1}(>15)\right]$. Los valores son medias \pm error estándar. En el Ensayo en macetas, letras diferentes indican diferencias significativas entre tratamientos y horas $(P<0.05)$.

Table 1. Red:far red ratio (R:RL) in the pot experiment. Measurements were made along the day, in plants exposed to full sun (sol) or bellow a dense canopy coverage (canopeo). In the gap experiment in the rainforest, R:RL was measured at midday in each range of total transmitted light, estimated with the hemispheric photographs [lower than 5 mol.m ${ }^{-2}$.day ${ }^{-1}(<5)$, between 5 and $10 \mathrm{~mol}^{-2} \mathrm{~m}^{-2}$ day ${ }^{-1}(5-10)$, between 10 and $15 \mathrm{~mol}^{-2} \mathrm{day}^{-1}(10-15)$ and higher than 15 mol. $\mathrm{m}^{-2}$.day $\left.{ }^{-1}(>15)\right]$. Data are means \pm standard error. In the pot experiment, different letters indicate significant differences between treatments and hours $(P<0.05)$.

\begin{tabular}{lcccccc}
\hline \multicolumn{5}{c}{ Ensayo en macetas } & \multicolumn{4}{c}{ Relación R:RL } \\
\hline Hora & Sol & Canopeo & $<5$ & $5-10$ & $10-15$ & $>15$ \\
9 & $0.99 \pm 0.01 \mathrm{e}$ & $0.51 \pm 0.01 \mathrm{a}$ & & & & \\
12 & $1.04 \pm 0.01 \mathrm{e}$ & $0.75 \pm 0.07 \mathrm{c}$ & $0.58 \pm 0.09 \mathrm{a}$ & $0.68 \pm 0.08 \mathrm{a}$ & $0.76 \pm 0.07 \mathrm{ab}$ & $0.96 \pm 0.10 \mathrm{~b}$ \\
14 & $0.99 \pm 0.01 \mathrm{e}$ & $0.52 \pm 0.06 \mathrm{a}$ & & & & \\
16 & $0.89 \pm 0.01 \mathrm{~d}$ & $0.64 \pm 0.01 \mathrm{~b}$ & & & & \\
\hline
\end{tabular}

El gradiente de cobertura generado en el ensayo de claros fue muy amplio. El porcentaje de canopeo abierto varió entre $1.2 \%$ en sitios con mayor cobertura y $32.4 \%$ en sitios con menor cobertura (Figura 1). En estos sitios, la luz transmitida total varió entre 0.6 y 18.7 mol. $\mathrm{m}^{-2}$.día ${ }^{-1}$, en relación con el porcentaje de

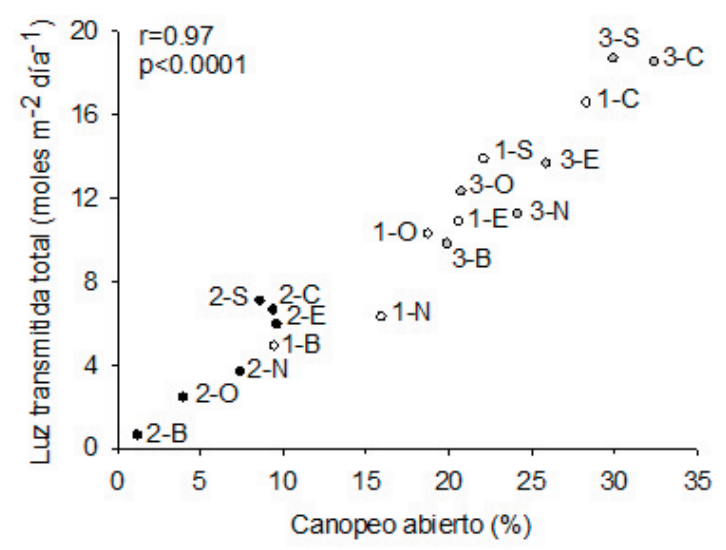

Figura 1. Correlación entre la luz transmitida total (mol.m ${ }^{2}$.día ${ }^{-1}$ ) y \% de canopeo abierto, medido en cada posición por claro para los 3 claros (ensayo en claros). Los números 1 a 3 indican el claro. Las letras indican la posición dentro del claro: $\mathrm{B}$ (debajo del bosque), $\mathrm{N}$ (norte), S (sur), E (este), $\mathrm{O}$ (oeste), C (centro). El tamaño de cada claro es: 1) $119 \mathrm{~m}^{2}$ (círculos grises oscuros), 2) $68 \mathrm{~m}^{2}$ (círculos negros) y 3) 172 $\mathrm{m}^{2}$ (círculos grises claros) de área extendida.

Figure 1. Correlations between total transmitted light $\left(\right.$ mol.m ${ }^{-2}$.day $\left.{ }^{-1}\right)$ and 5 of open canopy in each position within the three gaps (gap experiment). Numbers from 1 to 3 indicate the number of the gap. Letters indicate the position in the gap: B (below the canopy), N (North), $\mathrm{S}$ (South), E (East), O (West), C (center of the gap). The size of each gap is: 1) $119 \mathrm{~m}^{2}$ (dark grey circles), 2) 68 $\mathrm{m}^{2}$ (black circles) and 3) $172 \mathrm{~m}^{2}$ (light grey circles) of extended area. canopeo abierto (Figura 1). La luz transmitida total a cielo abierto fue $37.65 \mathrm{~mol} \cdot \mathrm{m}^{-2}$.día ${ }^{-1}$, por lo que el porcentaje de luz transmitida total para las diferentes posiciones del claro varió entre 1.6 y $50 \%$ de luz con respecto al cielo abierto. En sitios con menor cobertura, con luz transmitida total mayor a $15 \mathrm{~mol} \cdot \mathrm{m}^{-2}$. día ${ }^{-1}$ (>15) se registraron relaciones R:RL cercanas a los valores registrados para el tratamiento sol pleno del ensayo en macetas (Tabla 1). En los sitios donde la cobertura fue mayor, los valores de R:RL registrados fueron inferiores a los registrados en la condición de canopeo del ensayo en macetas (Tabla 1).

Tabla 2. Supervivencia de la parte aérea (\%) de las plantas de cancharana en los 2 ensayos [en macetas (sol, canopeo y shock lumínico) y ensayo en claros]. En el ensayo en macetas, la supervivencia se registró a los 6, 12 y 18 meses. Las plantas del tratamiento shock lumínico recibieron el tratamiento a los 12 meses de iniciado el ensayo. La supervivencia en los claros se registró a los 6, 12, 18, 36 y 44 meses desde la instalación de los ensayos.

Table 2. Above-ground survival (\%) of cancharana in both experiments. In the pot experiment, survival was registered at 6,12 and 18 months after planting, in full sun (sol), below the canopy (canopeo) and after a sudden change from canopy to full sun (shock lumínico). Shock lumínico was applied 12 months after planting. In the gap experiment (ensayo en claros), survival was registered 6, $12,18,36$ and 44 months after planting.

\begin{tabular}{lcccc}
\hline \multicolumn{5}{c}{ Supervivencia de la parte aérea (\%) } \\
& & Ensayo en macetas & Ensayo \\
& Sol & Canopeo & $\begin{array}{c}\text { Shock } \\
\text { lumínico }\end{array}$ & $\begin{array}{c}\text { ch } \\
\text { claros }\end{array}$ \\
\hline 6 meses & 94 & 96 & - & 91 \\
12 meses & 0 & 96 & - & 89 \\
18 meses & 62 & 96 & 100 & 82 \\
36 meses & - & - & - & 77 \\
44 meses & - & - & - & 70 \\
\hline
\end{tabular}




\section{Supervivencia y crecimiento}

Transcurridoelprimerperíododecrecimiento (6 meses), la supervivencia de las plantas de cancharana en las diferentes condiciones de cobertura establecidas en ambos ensayos fue mayor al 90\% (Tabla 2). En el ensayo de macetas, durante el invierno siguiente, las plantas que estaban a sol pleno sufrieron daño por heladas registrándose la muerte de la parte aérea del 100\% de las plantas (Tabla 2). Sin embargo, durante la primavera, el $62 \%$ rebrotó desde la base y sobrevivieron al finalizar el verano (Tabla 2). El 100\% de las plantas de cancharana que fueron sometidas al shock lumínico en la primavera de 2013 (12 meses) sobrevivieron transcurrido el verano (18 meses) (Tabla 2).

Las plantas de canopeo del ensayo en macetas crecieron significativamente más en altura que las plantas de sol $(29 \pm 1$ vs. $21 \pm 1 \mathrm{~cm}, \mathrm{~F}=23.7$, $P<0.0001)$ durante los primeros 6 meses. Sin embargo, el peso seco total de las plantas de sol fue mayor que las de canopeo (Figura 2).

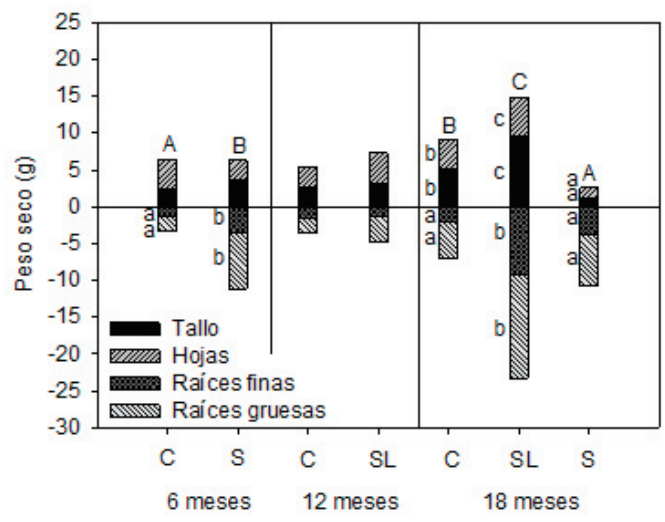

Figura 2. Peso seco total (g) y partición de materia seca (tallo, hojas, raíces finas y gruesas) de plantas de cancharana a los 6, 12 y 18 meses de iniciado el ensayo (ensayo en macetas). Las plantas crecieron bajo canopeo (C), a sol pleno (S) o fueron expuestas a un shock lumínico (SL) mediante un cambio abrupto de cobertura, de canopeo a sol pleno. Los valores negativos indican el peso seco de raíces. Letras minúsculas diferentes indican diferencias significativas entre tratamiento para cada compartimento, en cada fecha $(P<0.05)$. Las letras mayúsculas indican diferencias en el peso seco total.

Figure 2. Total dry mass (g) and dry mass partitioning (stem, leaves, fine roots and coarse roots) in cancharana plants 6, 12 and 18 months after experiment beginning (pot experiment). Plants grew below the forest canopy (C), under full sun (S) or they were exposed to a sudden light shock (SL) given by a coverage change from below the canopy to full sun. Negative numbers are root dry mass. Different lowercase letters indicate significant differences between treatments for each compartment, in each sampling date $(P<0.05)$. Different capital letters indicate differences in total dry mass.
Las plantas de cancharana que en la primavera fueron sometidas al cambio de cobertura (shock lumínico) respondieron de manera positiva: en 6 meses duplicaron su peso seco. La altura total no varió entre las plantas de canopeo denso y shock lumínico $(38 \pm 3$ y $35 \pm 2$ $\mathrm{cm}$, respectivamente), aunque la altura fue significativamente mayor que la de las plantas de sol $(14 \pm 1, F=78.3, P<0.0001)$. Las plantas de canopeo invirtieron proporcionalmente mayor cantidad de materia seca a hojas (24\%) con respecto al peso seco total, en comparación con las plantas de sol y de shock lumínico (10 y $14 \%$, respectivamente) (Figura 2, Anexo, Tabla 1A). Las plantas del shock lumínico acumularon significativamente más materia seca a las raíces y al tallo (Figura 2).

En el ensayo en claros, el PAR registrado al mediodía varió entre 3 y más de 2000 mol.m ${ }^{2} . \mathrm{s}^{-1}$ en las diferentes posiciones. A los 44 meses se registraron 48 plantas muertas en total, de las cuales el 71\% murió en las situaciones de mayor cobertura (menos de $200 \mu \mathrm{mol} . \mathrm{m}^{-2} \cdot \mathrm{s}^{-1}$ de PAR al mediodía) (Figura 3).

Las plantas de cancharana partieron inicialmente con la misma altura; la altura de las plantas que crecieron con luz $<5$ mol.m

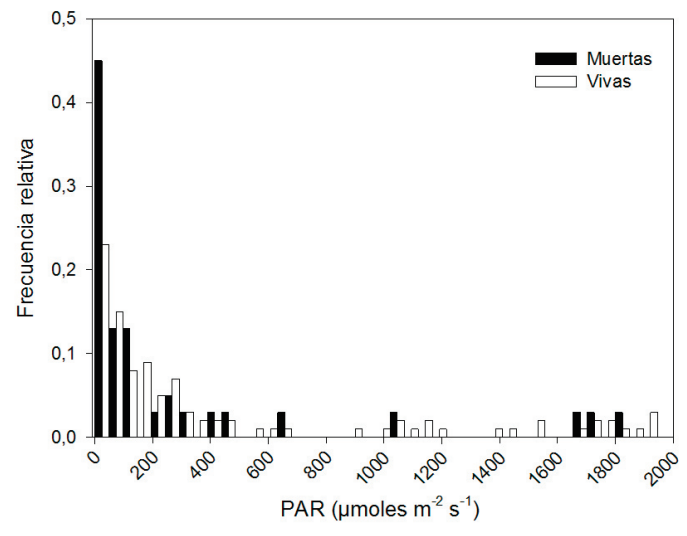

Figura 3. Frecuencia relativa para cada clase de PAR (intensidad de radiación fotosintéticamente activa al mediodía) de plantas vivas (barras grises) y muertas (barras negras) a los 44 meses de realizada la plantación en el ensayo en claros. Para esa fecha, de las 162 plantas iniciales, 124 estaban vivas y 38 muertas. El $45 \%$ de las plantas muertas estaba en la clase más baja de PAR. La distribución de las plantas vivas fue más pareja en todas las clases de PAR.

Figure 3. Relative frequency of alive plants (grey columns) and dead plants (black columns) 44 months after planting, in each range of photosynthetic active radiation (PAR) at midday (gap experiment). In that date, from the 162 plants, 124 were alive and 38 dead. $45 \%$ of the dead plants was at the lowest class, while the distribution of live plants was more even along classes. 
Tabla 3. Parámetros de las regresiones en dos tramos y sus significancias ( $P$-valor) de la altura $(\mathrm{cm})$ a lo largo del tiempo (meses) para las 4 categorías de luz transmitida: menor a $5 \mathrm{~mol} \cdot \mathrm{m}^{-2} \cdot$ día $^{-1}(<5)$, entre 5 y $10 \mathrm{~mol} \cdot \mathrm{m}^{-2}$.día $\mathrm{a}^{-1}$ (5-10), entre 10 y 15 mol.m ${ }^{-2}$.día ${ }^{-1}(10-15)$ y mayor a 15 mol.m $\mathrm{m}^{-2}$.día ${ }^{-1}(>15)$. $\alpha$ es intersección en el eje y; $\beta 1$ es la pendiente del primer tramo; $\gamma$ es el punto de inflexión y $\beta 2$ es la pendiente del segundo tramo. Las regresiones se observan en la Figura 5.

Table 3. Two-piecewise regression parameters and p-values between height $(\mathrm{cm})$ along the time (months) for each of the four transmitted light categories: lower than 5 mol.m ${ }^{-2}$.day ${ }^{-1}(<5)$, between 5 and $10 \mathrm{~mol}^{-2}$.day ${ }^{-1}(5-10)$, between 10 and $15 \mathrm{~mol} \cdot \mathrm{m}^{-2}$.day ${ }^{-1}(10-15)$ and higher than $15 \mathrm{~mol} \cdot \mathrm{m}^{-2}$. day ${ }^{-1}(>15) . \alpha$ is the interception in axe $y ; \beta 1$ is the slope of the first segment; $\gamma$ is the inflexion point and $\beta 2$ is the slope of the second segment. Regressions are plotted in Figure 5.

\begin{tabular}{ccccccccc}
\hline \multicolumn{2}{l}{ Intensidad de luz transmitida $\left(\mathrm{mol}^{-2} \mathrm{~m}^{-2}\right.$.ía $\left.^{-1}\right)$} & & & & & \\
& $<5$ & $P$-valor & $5-10$ & $P$-valor & $10-15$ & $P$-valor & $>15$ & $P$-valor \\
\hline$\alpha$ & 13.64 & $<0.01$ & 12.44 & $<0.01$ & 11.47 & 0.05 & 12.81 & 0.01 \\
$\beta 1$ & 0.1 & 0.87 & 1.89 & $<0.01$ & 2.37 & 0.03 & 2.44 & 0.01 \\
$\gamma$ & 3.78 & 0.29 & 20 & $<0.01$ & 11.64 & 0.75 & 10.62 & $<0.01$ \\
$\beta 2$ & 0.62 & $<0.01$ & 1.04 & $<0.01$ & 2.6 & $<0.01$ & 4.88 & $<0.01$ \\
\hline
\end{tabular}

${ }^{2}$.día ${ }^{-1}$ se mantuvo similar durante los 44 meses posteriores y tuvieron una tasa de crecimiento mucho menor que las plantas que crecieron en las otras tres categorías de luz (luz 5-10, luz 10-15 y luz >15), tal como se evidencia en la pendiente del primer tramo ( $\beta 1$ ) (Figura 4, Figura 5, Tabla 3). Pasado el primer año luego de la plantación, el crecimiento fue mayor a medida que la disponibilidad de luz fue mayor, tal como lo indica la pendiente del segundo tramo ( $\beta 2$, Tala 3). La altura de las plantas de cancharana fue mayor cuando la luz transmitida total fue superior a $15 \mathrm{~mol} . \mathrm{m}^{-}$ ${ }^{2}$. día ${ }^{-1}$. Las diferencias anteriores se reflejan en la menor pendiente del segundo tramo $(\beta 2)$ para las plantas que crecieron con luz $<5$ con

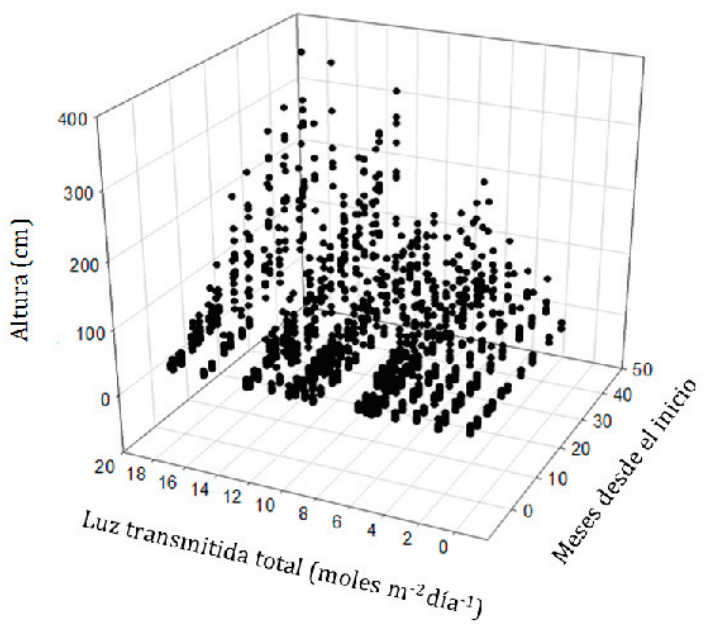

Figura 4. Altura $(\mathrm{cm})$ de las plantas de cancharana a lo largo de los 44 meses (meses de crecimiento) en las diferentes condiciones de luz recibida a lo largo del año (luz transmitida total, mol.m ${ }^{-2}$.día ${ }^{-1}$ ) del ensayo de los claros.

Figure 4. Plant height $(\mathrm{cm})$ along the first 44 months after planting, respect to mean total incident radiation along the year $\left(\mathrm{mol} . \mathrm{m}^{-2}\right.$.day $\left.{ }^{-1}\right)$ in the gap experiment.

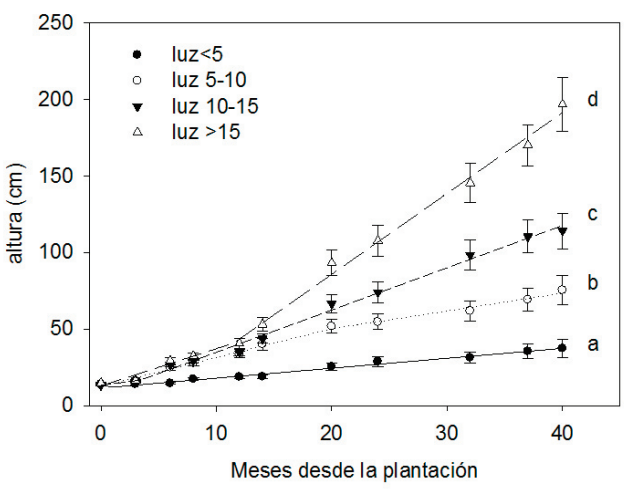

Figura 5. Regresiones en dos tramos entre la altura (cm) y el tiempo desde la plantación (meses) de plantas de cancharana en diferentes posiciones de los claros (experimento en claros). Cada curva representa un rango de luz transmitida total: menor a $5 \mathrm{~mol} \cdot \mathrm{m}^{-2}$.día ${ }^{-1}(<5)$, entre 5 y 10 mol.m ${ }^{-2} \cdot$ día $^{-1}$ (5-10), entre 10 y 15 mol.m ${ }^{-2}$.día ${ }^{-1}(10-15)$ y mayor a 15 mol.m $\mathrm{m}^{-2}$.día ${ }^{-1}(>15)$. Las barras en cada punto indican el error estándar.

Figure 5. Two-piecewise regression between plant height $(\mathrm{cm})$ and time from plantation (months) of cancharana plants in different positions in the gaps (gap experiment). Each curve represents a range of total transmitted light: lower than 5 mol.m $\mathrm{m}^{-2}$.día ${ }^{-1}(<5)$, between 5 and 10 mol.m ${ }^{2}$.día ${ }^{-1}(5-10)$, between 10 and $15 \mathrm{~mol} \cdot \mathrm{m}^{-2}$.día ${ }^{-1}(10-15)$ and higher than 15 mol.m ${ }^{-2}$.día ${ }^{-1}(>15)$. Bars in each point indicate standard error.

respecto a las que crecieron con luz $>15$ ( $\beta 2$ : 0.63 y $4.89 \mathrm{~cm} / \mathrm{mes}$, respectivamente) (Figura 5, Tabla 3).

\section{DISCUSIÓN}

La radiación es un factor clave para la supervivencia y el crecimiento de las plantas, y los niveles de cobertura del dosel modulan la cantidad de luz que llega a los diferentes microambientes en el bosque. En este estudio se evaluó la supervivencia y crecimiento de plantas de cancharana en ambientes contrastantes y en un gradiente 
lumínico amplio generado por claros en un bosque nativo secundario. Más del 90\% de las plantas de cancharana sobrevivieron a la primera estación de crecimiento en todos los ambientes de cobertura evaluados. En otros estudios con especies nativas de Misiones se reportaron valores similares de supervivencia de plantas que crecieron a cielo abierto y bajo una cubierta de pino (Crechi et al. 2005). Cancharana posee diferentes estrategias para aclimatarse a las distintas condiciones de cobertura y al cambio de la misma. Por un lado, a sol pleno, las plantas destinaron más cantidad de materia seca hacia las raíces; es posible que esta estrategia constituya una ventaja para abastecer de agua a las hojas y tallos que se encuentran en un ambiente con elevado déficit de saturación de la atmósfera y temperatura elevada (Figura 2). La misma respuesta se observó cuando las plantas fueron expuestas súbitamente a sol pleno (tratamiento shock lumínico) luego de estar un año bajo canopeo. Por otro lado, las plantas que crecieron siempre con poca disponibilidad de luz (tratamiento canopeo) acumularon mayor materia seca en hojas y fueron más altas que las de sol pleno (Tabla 2, Figura 2). Los cambios morfológicos se producen en respuesta a los cambios en la cantidad y calidad de luz, principalmente la relación rojo:rojo lejano (Dalling et al. 2004), le permiten a la planta captar mayor cantidad de luz y aumenta la probabilidad de interceptar flecos de luz de alta radiación que pueden llegar a los estratos inferiores del bosque con el movimiento de las hojas (Smith and Berry 2013).

A los 18 meses, la producción de materia seca de las plantas de canopeo fue menor que las plantas de shock lumínico, lo que indica que, a pesar de los cambios morfológicos, la baja radiación debajo del canopeo limitó la capacidad de fijar carbono y, por lo tanto, de crecer (Givnish 1988). En consecuencia, la estrategia en el cambio de distribución de materia seca le permite sobrevivir, pero no es suficiente para compensar la baja disponibilidad de luz. De esta manera, el crecimiento es menor que el de plantas expuestas a mayor radiación. En otro ensayo en la misma región, plantas de Cedrela fissilis Vell. (Meliaceae), una especie nativa de Misiones (de la misma familia botánica que la cancharana) respondieron de manera similar cuando fueron expuestas a diferentes condiciones de cobertura y al cambio de la disponibilidad de luz (Sanches et al. 2017). Estas respuestas de aclimatación nos indican que esta especie tiene la capacidad de aclimatarse en 6 meses a baja y alta radiación, pero luego necesita irradiancias altas para crecer. Esto se confirma en el ensayo de los claros, en el que las plantas expuestas a distinta disponibilidad de luz acrecientan su diferencia en la tasa de crecimiento en altura a medida que pasa el tiempo (Figura 5, Tabla 3).

Si bien la luz es uno de los factores abióticos más importantes para el establecimiento y crecimiento de las plantas, hay otros factores climáticos que los pueden condicionar. Las plantas de cancharana que crecieron a sol pleno fueron afectadas por las bajas temperaturas del invierno. Este daño por frío puede producirse por una acumulación de horas de temperaturas bajas, superiores a $0{ }^{\circ} \mathrm{C}$ (daño por frío), o por heladas con temperaturas menores a $0{ }^{\circ} \mathrm{C}$ (congelamiento) (Larcher 2005). Durante el invierno de 2013 (post plantación), las temperaturas mínimas absolutas mensuales fueron de $3{ }^{\circ} \mathrm{C},-3{ }^{\circ} \mathrm{C}, \mathrm{y}-2.5{ }^{\circ} \mathrm{C}$ para junio, julio y agosto, respectivamente (datos de la estación meteorológica del sitio donde se instaló el ensayo). Por otro lado, en los registros climáticos de los últimos 30 años se han reportado mínimas absolutas mensuales para los meses de invierno de entre $8^{\circ} \mathrm{C}$ y $-7{ }^{\circ} \mathrm{C}$. Por lo tanto, es posible que ocurran heladas de corta duración en esta zona. Durante el invierno, el 100\% de la parte aérea de las plantas de cancharana que estaban sin cobertura de dosel murieron, posiblemente a causa de las temperaturas bajo cero ocurridas. Sin embargo, durante la primavera siguiente, el 68\% de las plantas rebrotaron desde la base. En otro trabajo, también las temperaturas bajo cero produjeron la muerte de un $10 \%$ de las plantas de Enterolobium contortisiliquum (Vell) Morong (timbó) establecidas a cielo abierto y causaron daños en la parte aérea de los árboles que sobrevivieron (Crechi et al. 2005). Por otro lado, las plantas de timbó establecidas bajo cubierta de pino no registraron daños por temperaturas $<0{ }^{\circ} \mathrm{C}$ durante los 2 primeros años (Crechi et al. 2005). De manera similar, en nuestros ensayos, las plantas de cancharana que crecieron en los claros o bajo cobertura de canopeo (ensayo de macetas), no evidenciaron síntomas de daños por temperaturas bajo cero, durante el primer invierno post trasplante (Tabla 2). El gradiente de cobertura generado por los claros garantizó el establecimiento y supervivencia durante el primer invierno de las plantas de cancharana, aun en el centro del claro 3, que fue el de mayor superficie (Tabla 2) $\mathrm{y}$, por lo tanto, fue el microambiente con mayor 
exposición a las bajas temperaturas. Además, el efecto amortiguador del claro permitió la supervivencia y crecimiento de las plantas en los años posteriores. Esto nos indicaría que durante las primeras etapas de crecimiento, cancharana necesita cobertura para que no sufrir daños por frío o congelamiento durante el invierno. Muchas veces, estos requerimientos no son considerados cuando sólo se clasifican a las plantas en base a su respuesta a la luz. Los requerimientos de cobertura durante los meses fríos deben ser considerados en las selvas subtropicales, en las que las temperaturas invernales por debajo de cero ocurren casi todos los años.

Las características microambientales de los claros dependerán de su tamaño y de otros factores como, por ejemplo, la orientación respecto de los puntos cardinales, la densidad de copas (determinada por las especies y la altura de los árboles que forman el borde del claro) y la vegetación que crece en su interior. Las condiciones ambientales del sotobosque del claro favorecen la colonización de especies pioneras arbóreas, arbustivas y principalmente herbáceas de ciclos cortos, que crecen rápidamente, cubren el espacio y generan una gran competencia por recursos (Tabarelli and Mantovani 1997; Muñoz et al. 2012). En este estudio se observó una asociación positiva entre el porcentaje de apertura y la luz transmitida. Sin embargo, hay sitios donde, para porcentajes de apertura similares, la luz incidente es distinta (Figura 1) debido a la estructura y al tamaño de los árboles que conforman el borde del claro. En los sitios con mayor cobertura, el PAR al mediodía varió entre 4 y de $200 \mu \mathrm{mol} \cdot \mathrm{m}^{-2} \cdot \mathrm{s}^{-1}$ y la relación R:RL fue $\sim 0.5$. Esta baja relación R:RL es esperable en ambientes con mayor cobertura, ya que el follaje absorbe una cantidad de luz roja al atravesar el dosel, antes de llegar al sotobosque. Valladares et al. (2012) encontraron valores similares de relación R:RL en el sotobosque de bosque secundarios templados. Los valores de R: RL que se registraron en algunos ambientes generados por el claro fueron menores que los medidos en la condición de canopeo denso del primer ensayo (Tabla 1). Esto puede deberse a la gran competencia que se genera en el sotobosque del claro, donde las plantas pioneras arbóreas y herbáceas dominaron rápidamente el sotobosque y la competencia por luz fue mayor. Esto explicaría por qué en estos sitios se registró la mayor frecuencia de individuos muertos y el menor crecimiento en altura, incluso menos que en la condición de canopeo del primer ensayo (Figura 3, Figura 5). En otro estudio, la supervivencia a los 2 años también fue menor en los sitios con mayor cobertura, para 2 especies con requerimientos contrastantes de luz (una demandante de luz y otra tolerante a la sombra) (Lu et al. 2018). Además, en el mismo trabajo, la especie demandante de luz creció más en altura, con menor cobertura. Esto podría deberse a que las especies forestales en las etapas juveniles tienen la capacidad de establecerse bajo el canopeo denso, pero luego de un tiempo necesitan mayor disponibilidad de luz para crecer (Lamprecht 1990).

La supervivencia y el crecimiento de las plantas de cancharana fue mayor en sitios del claro donde la cobertura era menor y la disponibilidad de luz, mayor (Figura 4). La tasa de crecimiento se duplicó luego de un año para las plantas que crecieron con más de 15 mol. $\mathrm{m}^{-2}$.día ${ }^{-1}$; esto se ve en la pendiente de la recta del segundo tramo, que es $4.8 \mathrm{~cm} / \mathrm{mes}$, el doble que la pendiente del primer tramo (Tabla 3). Algunos individuos con esta disponibilidad de luz alcanzaron $3.6 \mathrm{~m}$ de altura a los 44 meses (Figura 4). El porcentaje de luz trasmitida total es de aproximadamente $50 \%$ para la categoría de luz mayor a 15 mol.m ${ }^{2}$.día ${ }^{-1}$, lo que significa que estaría llegando al centro del claro más grande alrededor del 50\% de la luz transmitida total. En el otro extremo, las plantas que crecieron durante 4 años con intensidad de luz menor a $5 \mathrm{~mol} \cdot \mathrm{m}^{-2}$.día ${ }^{-1}$ tuvieron tasas de crecimiento bajas $(0.6 \mathrm{~cm} /$ mes). Otras especies tropicales con diferentes requerimientos de luz también crecieron más en altura con mayor disponibilidad de luz (Chou et al. 2017); esto es esperable especies de la sucesión intermedia, como la cancharana. La supervivencia en los claros a los 44 meses fue de $70 \%$, a pesar de la alta heterogeneidad de respuesta en crecimiento. Si bien la cancharana logra establecerse bajo el canopeo, luego de un año necesita ser liberada de competencia lumínica para poder crecer a una tasa mayor. Durante el primer año luego de la plantación, las tasas de crecimiento en las situaciones intermedias de luz son similares a la de mayor radiación, con tasas cercanas a $2 \mathrm{~cm} /$ mes. Sin embargo, luego de ese período, la velocidad de crecimiento se duplica a medida que se pasa del rango más bajo al siguiente de disponibilidad de luz (Tabla 3). Esto demuestra que luego de un año, el crecimiento en altura resulta muy estimulado por la luz. Consistentemente, 
la mayor cantidad de plantas muertas se encontraban en las posiciones con menor disponibilidad de luz (Figura 3). En un estudio con Dipteryx micrantha Harms, descripta como dependiente de los claros, las plantas crecieron más en altura en los sitios del claro donde la disponibilidad de luz era mayor, y también presentaron una elevada heterogeneidad de crecimiento según su posición en los claros (Ashton et al. 1995; Reátegui 2005).

La notable plasticidad morfológica de cancharana le permite aclimatarse en pocos meses a la condición de cobertura (Figura 2) y a la heterogeneidad de ambientes en el bosque (Figura 1); esto beneficia el establecimiento y la supervivencia de una elevada cantidad de plantas y una respuesta heterogénea en el crecimiento. No obstante, los sitios con mayor disponibilidad de luz garantizaron el mayor crecimiento de esta especie y evitaron el daño por frío durante el invierno.

Para responder a las preguntas de investigación planteadas podemos resumir que las plantas de cancharana lograron establecerse inicialmente en condiciones de cobertura muy contrastantes y en un gradiente muy amplio de luz. La supervivencia será elevada en todos los microambientes, pero el crecimiento será afectado por el microambiente: a mayor radiación, mayor crecimiento.

Para elegir los sitios donde se plantará cancharana hay que tener en cuenta que durante las primeras etapas de crecimiento, las plantas necesitan protección contra el frío durante el invierno, ya que las temperaturas bajo cero afectan la supervivencia de las plantas y disminuyen el crecimiento de la parte aérea. Por lo tanto, en áreas con riesgo de heladas se debería evitar plantar cancharana en áreas sin cobertura o en el centro de claros muy grandes. Si se quiere utilizar cancharana en planes de restauración en áreas abiertas sería recomendable brindarle una cobertura mínima para garantizar la supervivencia en los primeros inviernos. Sin embargo, las plantas pueden sobrevivir bien los veranos con elevada radiación y demanda evapotranspirativa; es decir, no sufren estrés hídrico ni lumínico, tal como habíamos propuesto en la hipótesis de trabajo en base a la información disponible en la bibliografía. Por otro lado, se podría plantar cancharana en sitios de bosques degradados donde la cobertura de sotobosque sea densa. Sin embargo, una vez que la planta está establecida (i.e., 1 año después de la plantación), la exposición a mayor luz favorece marcadamente el crecimiento, aunque las plantas sobreviven al menos 3 años bajo el canopeo denso, con muy baja disponibilidad de luz. Por lo tanto, sería recomendable realizar control del sotobosque cerca de las plantas entre un año y tres de realizada la plantación.

El cambio abrupto de cobertura, de sombra a mayor radiación, tendrá un efecto positivo en el crecimiento, ya que la cancharana se puede aclimatar en 2 ó 3 meses a un nuevo microambiente, ya sea que el cambio en el ambiente se haya producido porque la planta fue llevada de vivero a campo o porque cambiaron las condiciones de cobertura en el dosel superior o en el sotobosque. Si se planta en primavera, las plantas llegarán al verano con la partición de materia seca adecuada para el sitio de plantación, por ejemplo, mayor destino de materia seca a raíces en situaciones de poca cobertura o mayor proporción de materia seca a hojas si la cobertura es espesa.

Cancharana es una especie que se puede aclimatar a condiciones muy diferentes. Se la podría emplear en planes de restauración o de enriquecimiento de áreas degradadas que presenten microambientes variados.

Agradecimientos. Agradecemos a Tomás de Hagen, Celina Bertoni, Martín Santiago, Mauro Bartolozzi, Valeria Bernardo, Maia Plaza Behry Manuel Billieres por su colaboración técnica en las mediciones a campo y al personal de campo del Campo Anexo Manuel Belgrano, San Antonio, dependiente de la EEA Montecarlo por la colaboración en la instalación y cuidado de los ensayos. El proyecto fue financiado por los PIA 12010 y 14031, de la UCAR-Ministerio de Agroindustria de la Nación.

\section{REFERENCIAS}

Aimi, S. C. 2014. Tecnologia de sementes e crescimento inicial de mudas de Cabralea canjerana (Vell.) Mart. Master Thesis (Mestrado em Engenharia Florestal). Santa Maria, Brasil. Universidade Federal de Santa Maria. Pp. 110.

Amir, A. A. 2012. Canopy gaps and the natural regeneration of Matang mangroves. Forest Ecology and Management 269:60-67.

Ashton, P. M. S., C. V. S. Gunatilleke, I. A. U. N. Gunatilleke, and S. Journal. 1995. Seedling Survival and Growth of Four Shorea Species in a Sri Lankan Rainforest. Journal of Tropical Ecology 11:263-279.

Di Bitetti, M., G. Placci, and L. A. Dietz. 2003. Una Visión de Biodiversidad para la Ecorregión del Bosque Atlántico 
del Alto Paraná: Diseño de Paisaje para la Conservación de la Biodiversidad y prioridades para las acciones de conservación. World Wildlife Fund, Washington.

Cargnelutti Filho, A., M. M. Araujo, E. Gasparin, and A. L. de Avila. 2012. Dimensionamento amostral para avaliação de altura e diâmetro de mudas de Cabralea canjerana. Ciência Rural 42:1204-1211.

Carvalho, P. E. R. 1996. Infuência da intensidade luminosa e do substrato no crescimento, no conteúdo de clorofila e na fotossíntese de Cabralea canjerana (Vell.) MART. Subsp. Canjerana, Calophyllum brasiliense CAMB. e Centrolobium robustum (Vell) MART. EX Benth., na fase juvenil. Tese (Doutorado) - Universidade Federal do Paraná, Curitiba. Pp. 157.

Casanoves, F., M. G. Balzarini, J. A. Di Rienzo, L. González, M. Tablada, and C. W. Robledo. 2012. InfoStat. User Manual. Córdoba. Argentina.

Chazdon, R. L., R. W. Pearcy, D. W. Lee, and N. Fetcher. 1996. Photosynthetic responses of tropical forest plants to contrasting light environments. Pp. 5-85 en S. S. Mulkey, R. L. Chazdon and A. P. Smith, editores. Tropical Forest Plant Ecophysiology.

Chou, C. B., L. O. Hedin, and S. W. Pacala. 2017. Functional groups, species and light interact with nutrient limitation during tropical rainforest sapling bottleneck. Journal of Ecology 106:157-167. DOI: 10.1111/1365-2745.12823.

Coates, K. D. 2002. Tree recruitment in gaps of various size, clearcuts and undisturbed mixed forest of interior British Columbia, Canada. Forest Ecology and Management 155:387-398.

Crechi, E., R. Fernández, C. Domecq, A. Hennig, and B. Eibl. 2005. Crecimiento inicial de 3 especies latifoliadas nativas de interés económico en Misiones (Cordia trichotoma (Vell.) Arrab. ex Steudel, Balfourodendron redelianum (Engl.) Engl, Enterolobium contortisiliquum (Vell.) Morong.). Yvyrareta 13:1-9.

Dalling, J. W., K. Winter, and S. P. Hubbell. 2004. Variation in growth responses of neotropical pioneers to simulated forest gaps. Functional Ecology 18:725-736.

Denslow, J. S. 1987. Tropical rainforest gaps and tree species diversity. Annual Review of Ecology and Systematics 18: 431-451. DOI: 10.1146/annurev.es.18.110187.002243

Dudley, S. A., and A. L. File. 2007. Kin recognition in an annual plant. Biology Letters 3:435-8.

Fitter, A., and R. Hay. 2002. Enviromental Physiology of plants. 3rd Ed. Academic Press. Pp. 397.

Frazer, G. W., C. D. Canham, and K. Lertzman. 1999. Gap Light Analyzer (GLA): Imaging software to extract canopy structure and gap light transmission indices from true-colour fisheye photographs, users manual and program documentation.

Garbarino, M., E. B. Mondino, E. Lingua, T. A. Nagel, V. Dukić, Z. Govedar, and R. Motta. 2012. Gap disturbances and regeneration patterns in a Bosnian old-growth forest: A multispectral remote sensing and ground-based approach. Annals of Forest Science 69:617-625.

Gasser, D., C. Messier, M. Beaudet, and M. J. Lechowicz. 2010. Sugar maple and yellow birch regeneration in response to canopy opening, liming and vegetation control in a temperate deciduous forest of Quebec. Forest Ecology and Management 259:2006-2014.

Givnish, T. J. 1988. Adaptation to Sun and Shade: a whole-plant perspective. Australian Journal of Plant Physiology 15:63-92.

González, P. A., and S. R. Barth. 2013. Estudio ecológico y silvicultural de Cordia trichotoma, Cabralea canjerana y Picrasma crenata. Su potencialidad en la diversificación productiva. URL: 64.76.123.202/site/forestacion/proyectos_fo en MAGPyA, editor. Jornadas PIAS 2013. MAGPyA, Buenos Aires, Argentina.

Lamprecht, H. 1990. Silvicultura en los trópicos. Los ecosistemas forestales en los bosques tropicales y sus especies arbóreas -posibilidades y métodos para un aprovechamiento sostenido. GTZ GmbH, Eschborn.

Larcher, W. 2005. Climatic Constraints Drive the Evolution of Low Temperature Resistance in Woody Plants. Journal of Agricultural Meteorology 61:189-202.

De Lima, R. A. F., and L. C. De Moura. 2008. Gap disturbance regime and composition in the Atlantic Montane Rain Forest: The influence of topography. Plant Ecology 197:239-253.

Lu, D., G. G. Wang, J. Zhang, Y. Fang, C. Zhu, and J. Zhu. 2018. Converting larch plantations to mixed stands: Effects of canopy treatment on the survival and growth of planted seedlings with contrasting shade tolerance. Forest Ecology and Management 409:19-28.

Montagnini, F., B. Eibl, R. Fernandez, and M. Brewer. 2006. Strategies for restoration of forest landscapes. Experiences from Misiones, Argentina. Páginas 2-14, Actas II Congreso Forestal Latinoamericano IUFRO. Talca, Chile.

Montagnini, F., and C. F. Jordan. 2005. Tropical Forest Ecology. The Basis of Conservation and Management. En G. Dieter Czeschlik (ed.). Tropical Forestry. The basis for conservation and management. 1st Ed. Springer Berlin Heidelberg. Pp. 295.

Montgomery, R. A., and R. L. Chazdon. 2002. Light gradient partitioning by tropical tree seedlings in the absence of canopy gaps. Oecologia 131:165-174.

Morales, L. V., B. Fuentealba, S. Sevillano, I. M. Gómez, C. M. Segovia Salcedo, D. Renison, D. Green, C. Aucca, and I. Hensen. 2018. Oportunidades para acercar la ciencia a la práctica de la restauración de bosques y arbustales de Polylepis I. Ecología Austral 28(1-bis):291-300.

Moscovich, F., C. Dummel, M. Pinazo, O. Knebel, and R. Alcaraz. 2010. Caracterización fitosociológica de una porción de bosque nativo misionero secundario , con intervención antrópica. Quebracho 18(1-2):24-36.

Muñoz, A. A., M. E. González, C. Celedón, and T. T. Veblen. 2012. Respuesta inicial de la regeneración arbórea luego de la floración y muerte de Chusquea culeou (Poaceae) en bosques andinos del centro-sur de Chile. Bosque (Valdivia) 
33:9-10.

Reátegui Romo, M. 2005. Efecto de la luz en el crecimiento de plántulas de Dipteryx micrantha Harms "shihuahuaco" trasplantadas a sotobosque, claros y plantaciones. Ecología Aplicada 4:1-8.

Ríos, R. C., F. Galvão, and G. R. Curcio. 2008. Variaciones estructurales de la vegetación arbórea en tres ambientes de una selva con araucaria en Misiones, Argentina. Floresta 38:743-756.

Sanches, M. C., J. Marzinek, N. G. Bragiola, and A. R. Terra Nascimento. 2017. Morpho-physiological responses in Cedrela fissilis Vell. submitted to changes in natural light conditions: implications for biomass accumulation. Trees - Structure and Function 31:215-227.

Schüssler, G. 2006. Dinâmica populacional e aspectos da regeneração natural de Cabralea canjerana (Vell.) Mart. (Meliaceae) em uma zona de contato entre as florestas ombrófilas montanas, RS. Universidade Federal do Rio Grande do Sul, Brazil.

Smith, W. K., and Z. C. Berry. 2013. Sunflecks? Tree Physiology 33:233-237.

Tabarelli, M., and W. Mantovani. 1997. Colonização de clareiras naturais na floresta atlântica no sudeste do Brasil. Revista Brasileira de Botânica 20:57-66.

Tortorelli, L., R. A. Stella, and J. R. Ottone. 2009. Maderas y bosques argentinos. Orientación Gráfica Editora. 2a ed. Buenos Aires, Argentina.

Valladares, F., I. Aranda, and D. Sánchez-Gómez. 2004. La luz como factor ecológico y evolutivo para las plantas y su interacción con el agua. Páginas 335-370 en F. Valladares (ed.). Ecología del bosque mediterráneo en un mundo cambiante. EGRAF S. A., Madrid, España.

Valladares, F., A. Saldaña, and E. Gianoli. 2012. Costs versus risks: Architectural changes with changing light quantity and quality in saplings of temperate rainforest trees of different shade tolerance. Austral Ecology 37:35-43.

Welden, C., S. Hewett, S. Hubbell, and R. Foster. 1991. Sapling survival, growth, and recruitment: relationship to canopy height in a neotropical forest. Ecology 72:35-50. DOI: 10.2307/1938900.

Wright, J. S., H. C. Muller-Landau, R. Condit, and S. P. Hubbell. 2003. Gap-dependent recruitment, realized vital rates , and size distributions of tropical trees. Ecology 84:3174-3185.

Yang, W., F. Liu, L. Zhou, S. Zhang, and S. An. 2011. Trade-offs between growth and survival of non-pioneer lightdemanding tree seedlings in tropical forest of Hainan Island , China. Journal of Tropical Ecology 27:611-620. 